\title{
Network-level analysis of metabolic regulation in the human red blood cell using random sampling and singular value decomposition Christian L Barrett ${ }^{1}$, Nathan D Price ${ }^{2}$ and Bernhard O Palsson*1
}

\author{
Address: ${ }^{1}$ Bioengineering Department, University of California - San Diego, 9500 Gilman Drive, La Jolla, California, 92093-0412, USA and \\ 2Institute for Systems Biology, 1441 North 34th Street, Seattle, WA, 98103, USA \\ Email: Christian L Barrett - cbarrett@bioeng.ucsd.edu; Nathan D Price - nprice@systemsbiology.org; Bernhard O Palsson* - palsson@ucsd.edu \\ * Corresponding author
}

Published: 13 March 2006

BMC Bioinformatics2006, 7:132 doi:10.1/86/1471-2105-7-132

This article is available from: http://www.biomedcentral.com/147/-2105/7//32

(c) 2006Barrett et al; licensee BioMed Central Ltd.

This is an Open Access article distributed under the terms of the Creative Commons Attribution License (http://creativecommons.org/licenses/by/2.0), which permits unrestricted use, distribution, and reproduction in any medium, provided the original work is properly cited.
Received: 13 August 2005

Accepted: 13 March 2006

\begin{abstract}
Background: Extreme pathways (ExPas) have been shown to be valuable for studying the functions and capabilities of metabolic networks through characterization of the null space of the stoichiometric matrix (S). Singular value decomposition (SVD) of the ExPa matrix $\mathbf{P}$ has previously been used to characterize the metabolic regulatory problem in the human red blood cell (hRBC) from a network perspective. The calculation of ExPas is NP-hard, and for genome-scale networks the computation of ExPas has proven to be infeasible. Therefore an alternative approach is needed to reveal regulatory properties of steady state solution spaces of genome-scale stoichiometric matrices.
\end{abstract}

Results: We show that the SVD of a matrix (W) formed of random samples from the steady-state solution space of the $h R B C$ metabolic network gives similar insights into the regulatory properties of the network as was obtained with SVD of $\mathbf{P}$. This new approach has two main advantages. First, it works with a direct representation of the shape of the metabolic solution space without the confounding factor of a non-uniform distribution of the extreme pathways and second, the SVD procedure can be applied to a very large number of samples, such as will be produced from genome-scale networks.

Conclusion: These results show that we are now in a position to study the network aspects of the regulatory problem in genome-scale metabolic networks through the use of random sampling.

Contact: palsson@ucsd.edu

\section{Background}

In recent years, chemically-detailed metabolic networks capable of simulating growth have been reconstructed at the genome-scale for nearly twenty organisms [1]. Any metabolic network can be represented as a stoichiometric matrix, and by assuming steady-state conditions one can compute the "space" in which all allowable steady-state flux distributions reside. This space can be spanned by a set of extreme pathways (ExPas) [2,3], and for a given metabolic network this set is unique, invariant and irreducible [4]. ExPas are so named because they form the edges of the steady-state solution space of the network and thus characterize its "extreme" functions. Mathematically, the ExPas are a set of conical basis vectors that forms a convex cone in high-dimensional space. In this manner, the extreme pathways circumscribe all of the possible steady- 
state flux distributions possible in the metabolic network. Biochemically, each ExPa corresponds to a steady-state flux distribution through the network, and is more than just a linear set of reactions linking substrate to product. Specifically, each ExPa describes the relative fluxes among a set of reactions needed for the conversion of substrate(s) into product(s), and does so by creating all byproducts needed to maintain systemic balances and by simultaneously maintaining all cofactor pools at steady state. The development of such network-based pathway definitions, including the elementary flux modes [5-7], has led to a systems view of network properties [8]. Genome-scale metabolic pathway analysis $[5,9,10]$ holds much promise for understanding fundamental aspects of network functionality, robustness, and regulation $[11,12]$, for metabolic engineering $[13,14]$, and for elucidating many other systems properties [12,15-23].

The number of ExPas for a reaction network grows exponentially with network size, limiting their use for the analysis of large networks. Because of this limitation, ExPas have mainly been used to analyze realistic, but small, networks such as the hRBC [22]. In further work [4,24], singular value decomposition (SVD) was applied to the ExPa matrices (P), resulting in two key insights. First, it was shown that SVD of $\mathbf{P}$ can compute the effective dimensionality of the regulatory problem from a network perspective. Second, it was shown that the 'eigenpathways' that correspond to the modes of the SVD identified key network branch points that can represent key control points (reactions) in the network. These points constitute a minimal set of reactions whose control sets the steady state flux state for the entire network, represented as a specific location in the steady-state solution space. SVD rank orders these key branch points in accordance with the shape of the solution space and thus rank orders the effectiveness by which the branch points can be used to move the network's flux distribution around in the solution space. These features were demonstrated by showing that the dominant features of the first five eigenpathways correspond to actual major points of control in the hRBC and can effectively locate the solution in a 5-dimensional space [24]. SVD of $\mathbf{P}$ can thus define the magnitude and nature of the regulatory problem associated with controlling a metabolic network.

Given the fundamental understanding of network properties that can be derived from the SVD of $\mathbf{P}$, it would be natural to perform the same analysis on the much larger genome-scale metabolic networks that have already been reconstructed. Unfortunately, this is not currently possible due to the NP-hard nature of calculating ExPas. Even if computing ExPas were possible on a genome-scale, the likely result would be matrices containing extremely large numbers of ExPas, many more than would be needed using uniform random sampling. This number of ExPas presents an additional formidable hurdle for the SVD calculation, which is an $\mathrm{O}\left(\mathrm{n}^{2}\right)$ algorithm [25].

In this work, we demonstrate an approach that addresses this problem. As mentioned above, the set of ExPas for a metabolic network defines the shape of the steady-state solution space of the network. The information that SVD of the set of ExPas provides is a description of the space. The eigenvectors that are produced by the SVD indicate, in rank order, the directions corresponding to the greatest variance in the high-dimensional space, and the singular values give the relative magnitude of this variation. A potentially better approach is to characterize the shape of the solution spaces by generating uniform random samples within it. Each sample solution is used as a column in a matrix (W). With enough samples, a "picture" of the solution space emerges, which can then be analyzed using SVD in the same manner as was done previously for the ExPas. In this work, we performed uniform random sampling of the steady-state solution space of the hRBC metabolic network and applied SVD to the matrix, $\mathrm{W}$, of these samples. We show that similar, though not identical, results are obtained through SVD of $\mathrm{W}$ as were obtained through SVD of P. Thus, this study provides a new method for the calculation of Principle Regulatory Modes (PRMs) that improves upon the previous method by being more tractable for genome-scale networks due to its independence from ExPas. Additionally, the interpretation of the results of the new method are more straightforward because it characterizes the shape of the solution space uniformly, without being affected by the non-uniformity of the distribution of ExPas on the edges of the solution space.

\section{Results}

\section{Ascertaining sampling sufficiency}

We first determined that the steady-state solution space of the hRBC was thoroughly sampled and that an accurate set of solutions had been sampled. Incomplete sampling would have the effect of misrepresenting the shape of the space and would lead to inaccurate results. We determined the thoroughness of the sampling by plotting the angle (see Methods section) between subsequent SVD modes for increasing sample sizes. This convergence is shown in Figure 1 for the five modes with the largest singular values. As can be seen, convergence occurred after using just a few thousand sample solutions, with very little change over the remainder of the one million samples. This result demonstrates that the solution space of a small metabolic network, which in the hRBC contains just 51 reactions, can be studied with relatively few samples. 

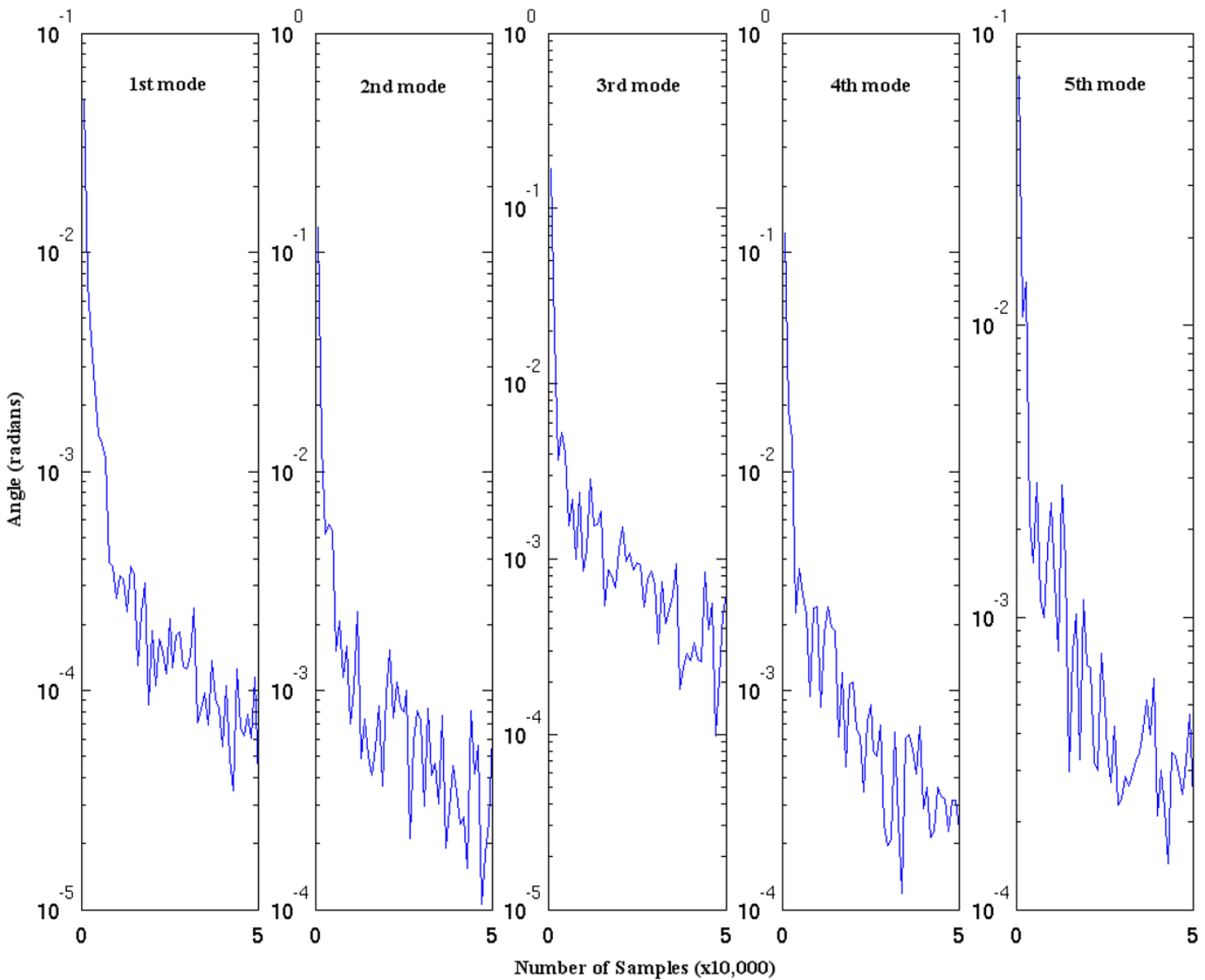

\section{Figure I}

The SVD procedure was applied for increasingly larger sample sizes (up to $1,000,000$ in increments of 2,000) and the first five modes at each sample size were recorded. These plots show the convergence of the angle between the modes for subsequent sample sizes.

\section{Comparing the modes derived from $P$ and $W$}

The modes corresponding to the first five singular values obtained from SVD of the extreme pathways (eigenpathways) and from the samples (principal regulatory modes, or PRMs) were shown on a flux map of the hRBC. These maps were then compared by placing them side-by-side in Figures 2, 3, 4, 5, 6. The differences and similarities between the eigenpathways and PRMs are addressed in the Discussion section.

\section{Assessing the correspondence of inferred reaction regulatory importance}

One of the primary insights resulting from the application of SVD to $\mathbf{P}$ was an understanding of the relative importance amongst all of the reactions in terms of regulatory control. The aim of this work was to show that similar insight can be obtained through uniform random sampling. To do this, we followed a previously developed procedure [26] and scored each reaction by the $l^{2}$-norm (length) of its loading vector, which is a vector of the reaction's contribution to each of the eigenvectors resulting from the SVD procedure (see Methods section). The rationale for this scoring method is based on our goal of identifying those reactions most critical for controlling flux through the metabolic network. These control reactions are expected to have larger contributions to the eigenvectors resulting from the SVD procedure. The impact of these larger contributions is reflected in a larger absolute value of the loading vector, signifying a larger distance of the reaction from the origin. So in this context the $l^{2}$-norm quantifying the length of the loading vector is appropriate for our purposes. 

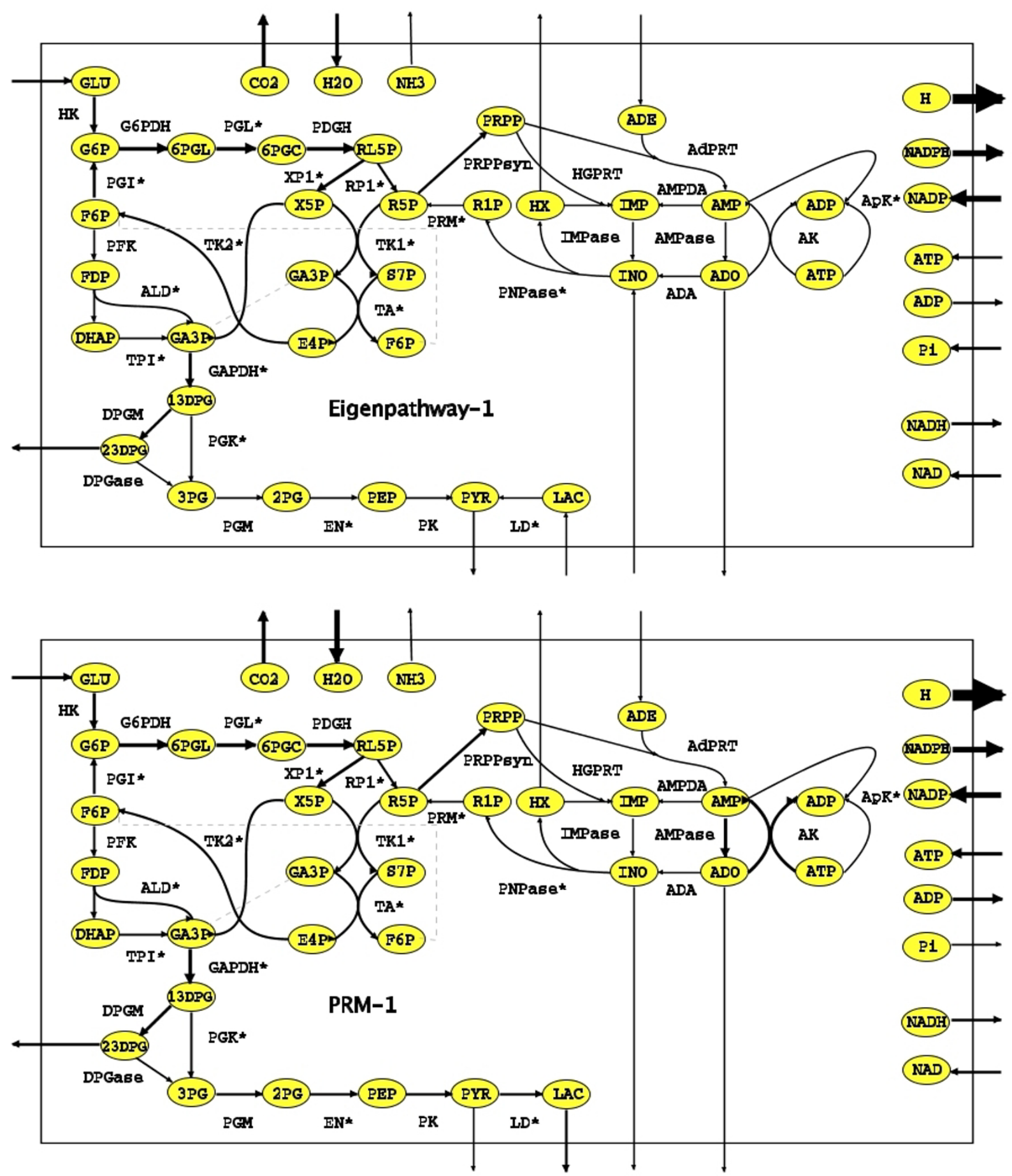

\section{Figure 2}

The first eigenpathway derived from SVD of the extreme pathways matrix and the first PRM derived from SVD of the samples matrix for the $\mathrm{hRBC}$ metabolic network. The relative values of the flux values is indicated by the thickness of the arrows between metabolites. The metabolite and reaction definitions are given in Table I and Table 2. 

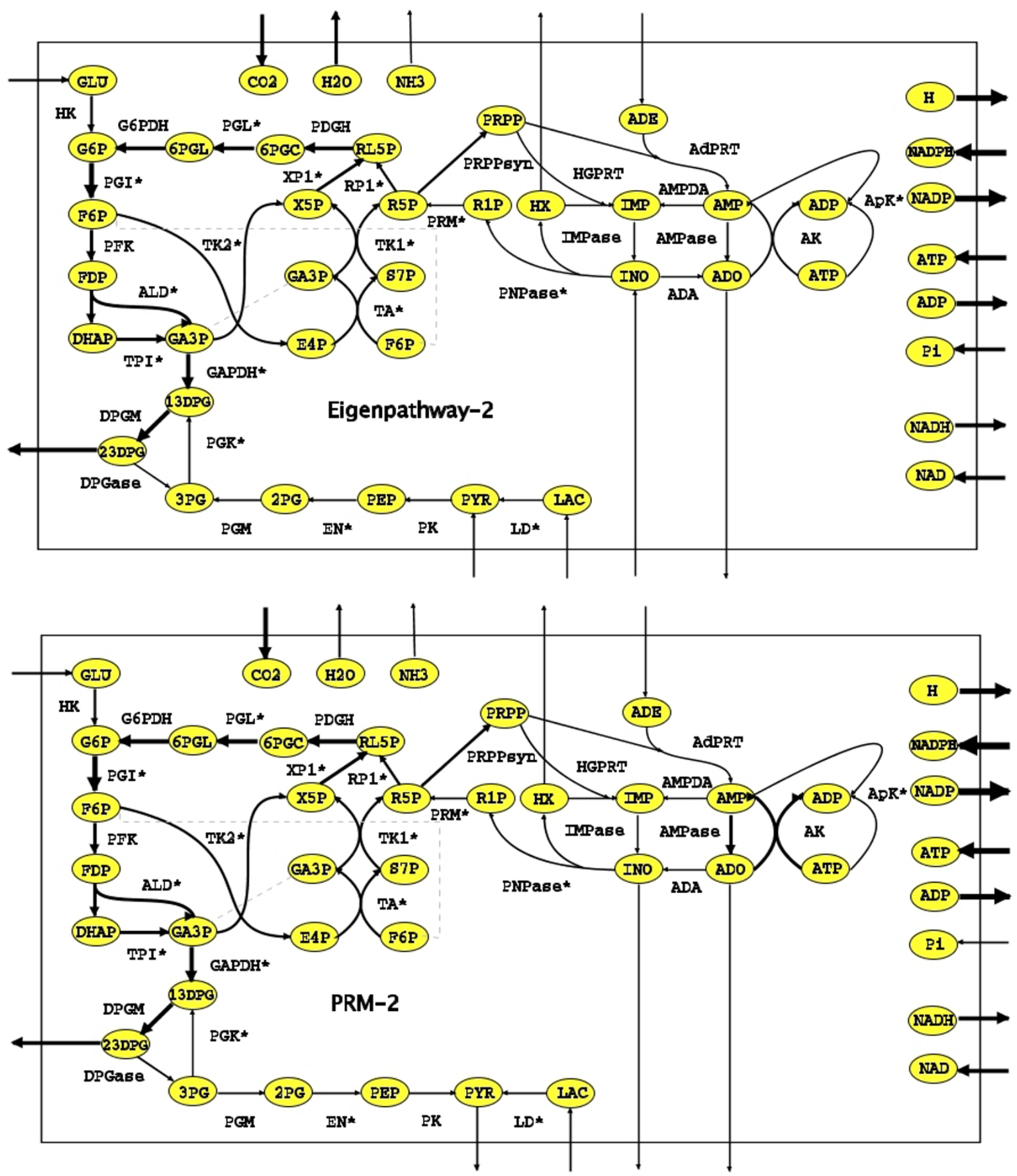

Figure 3

The second eigenpathway derived from SVD of the extreme pathways matrix and the second PRM derived from SVD of the samples matrix for the hRBC metabolic network. The relative values of the flux values is indicated by the thickness of the arrows between metabolites. The metabolite and reaction definitions are given in Table I and Table 2. 

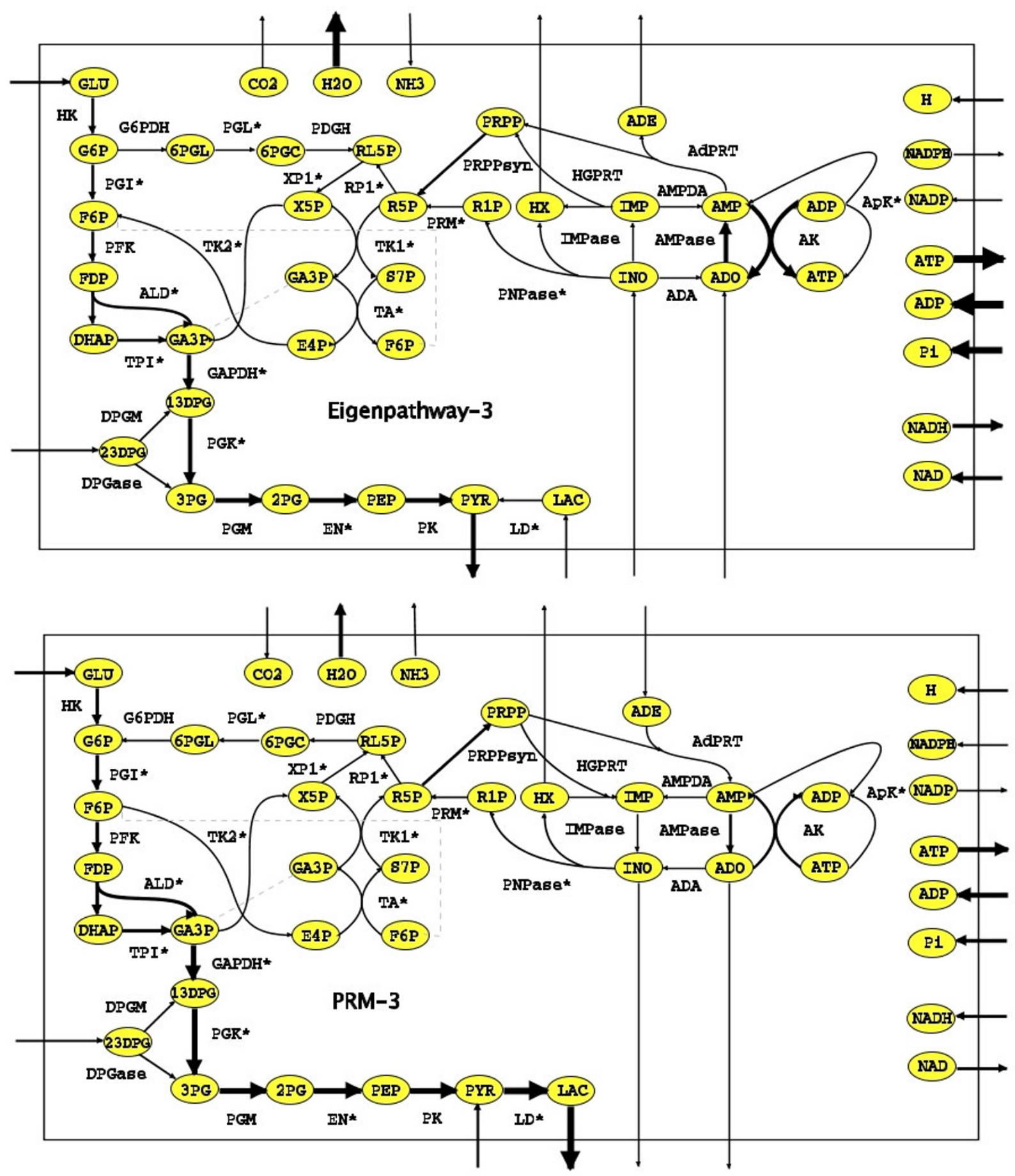

\section{Figure 4}

The third eigenpathway derived from SVD of the extreme pathways matrix and the third PRM derived from SVD of the samples matrix for the hRBC metabolic network. The relative values of the flux values is indicated by the thickness of the arrows between metabolites. The metabolite and reaction definitions are given in Table I and Table 2. 

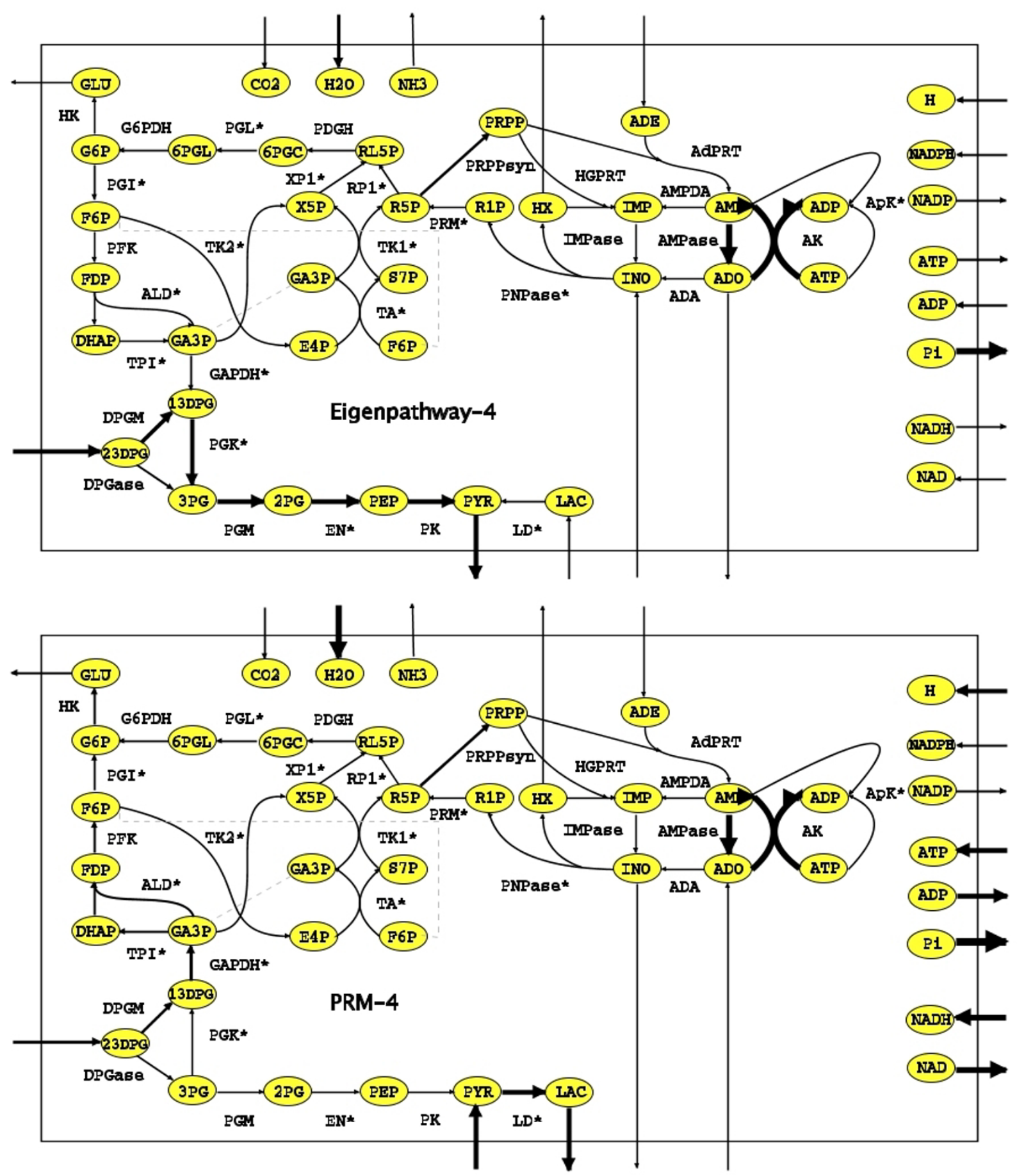

Figure 5

The fourth eigenpathway derived from SVD of the extreme pathways matrix and the fourth PRM derived from SVD of the samples matrix for the hRBC metabolic network. The relative values of the flux values is indicated by the thickness of the arrows between metabolites. The metabolite and reaction definitions are given in Table I and Table 2. 

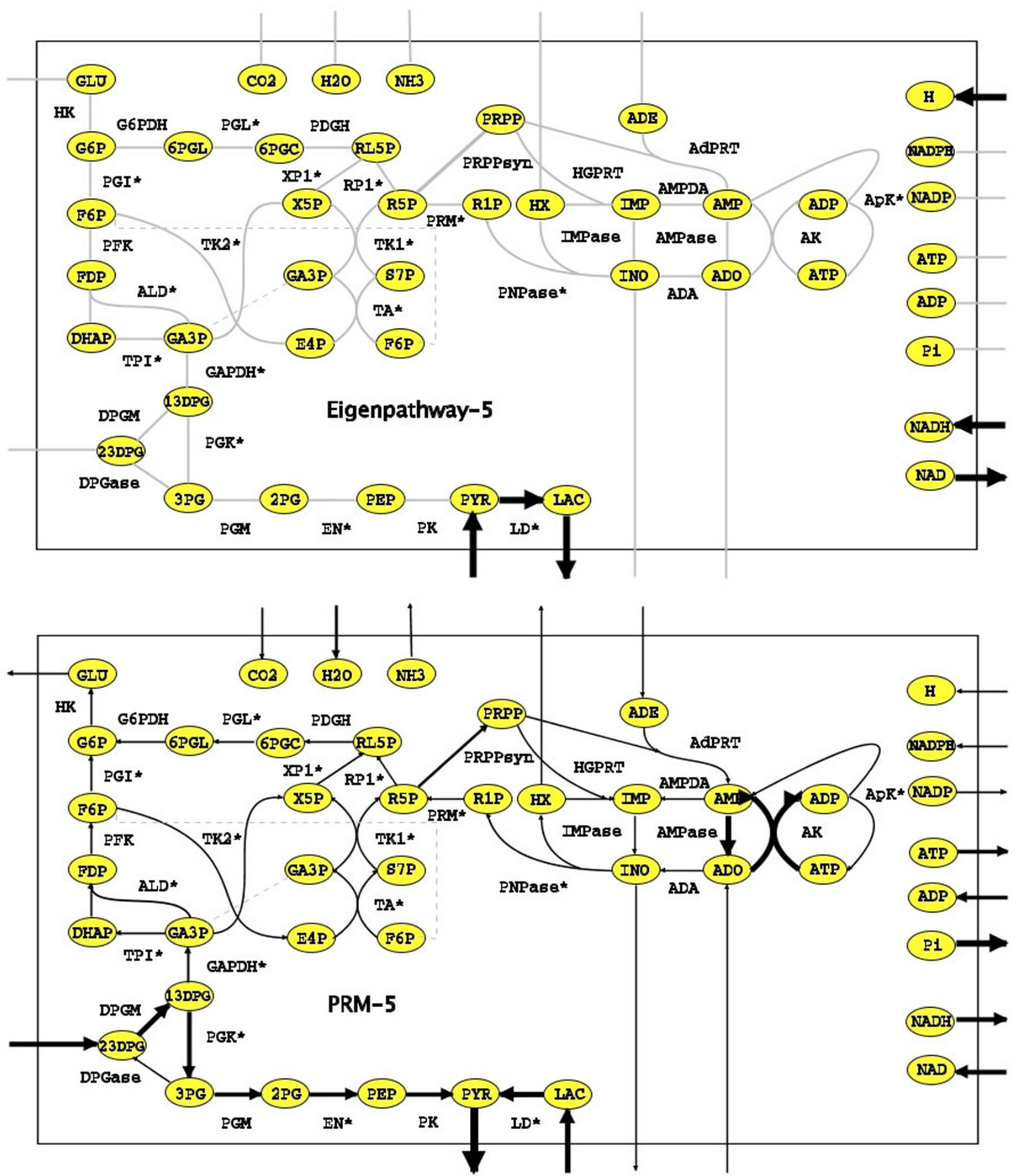

Figure 6

The fifth eigenpathway derived from SVD of the extreme pathways matrix and the fifth PRM derived from SVD of the samples matrix for the $\mathrm{hRBC}$ metabolic network. The relative values of the flux values is indicated by the thickness of the arrows between metabolites. The metabolite and reaction definitions are given in Table I and Table 2. 
Scores allow us to impart a rank ordering on the reactions. The degree to which the SVD of $\mathbf{W}$ and the SVD of $\mathbf{P}$ provide similar information about the regulatory importance of the reactions, then, is measured by the similarity in which they rank order the reactions by their scores. We measured this similarity by computing the rank correlation coefficient between the two rank orderings of reactions. Just like Pearson's correlation coefficient, the rank correlation has a range $[-1,1]$. We used two related rank correlation measures [27]: Kendall's tau and Spearman's rho. We found the reaction rankings to be highly correlated, resulting in a tau value of 0.88 with P-value $1.1^{*} 10^{-}$ 15 and a rho value of 0.97 with P-value $4.0^{*} 10^{-12}$. Based on these results, we claim that the sampling methodology produces similar insight into identifying the most important reactions for flux control as did the ExPa-based approach.

\section{Reconstruction of physiological flux distributions}

We determined how the modes computed from SVD of $\mathbf{W}$ were combined to reconstruct the physiological flux distributions and how the difference between the reconstructed and actual distributions diminished as more singular modes were used to reconstruct the desired solution. The reconstruction and difference computations were performed in the same manner as described previously (Price, Reed et al. 2003). Additionally, we assessed how the efficiency of these reconstructions compared with those based on the eigenpathways. The results are shown in Figure 7. These results indicate that in 3 out of the 4 cases, fewer of the PRMs are required for reconstructing the flux distributions than were required using eigenpathways. In the fourth case, the same number of modes yields similar reconstruction accuracy.
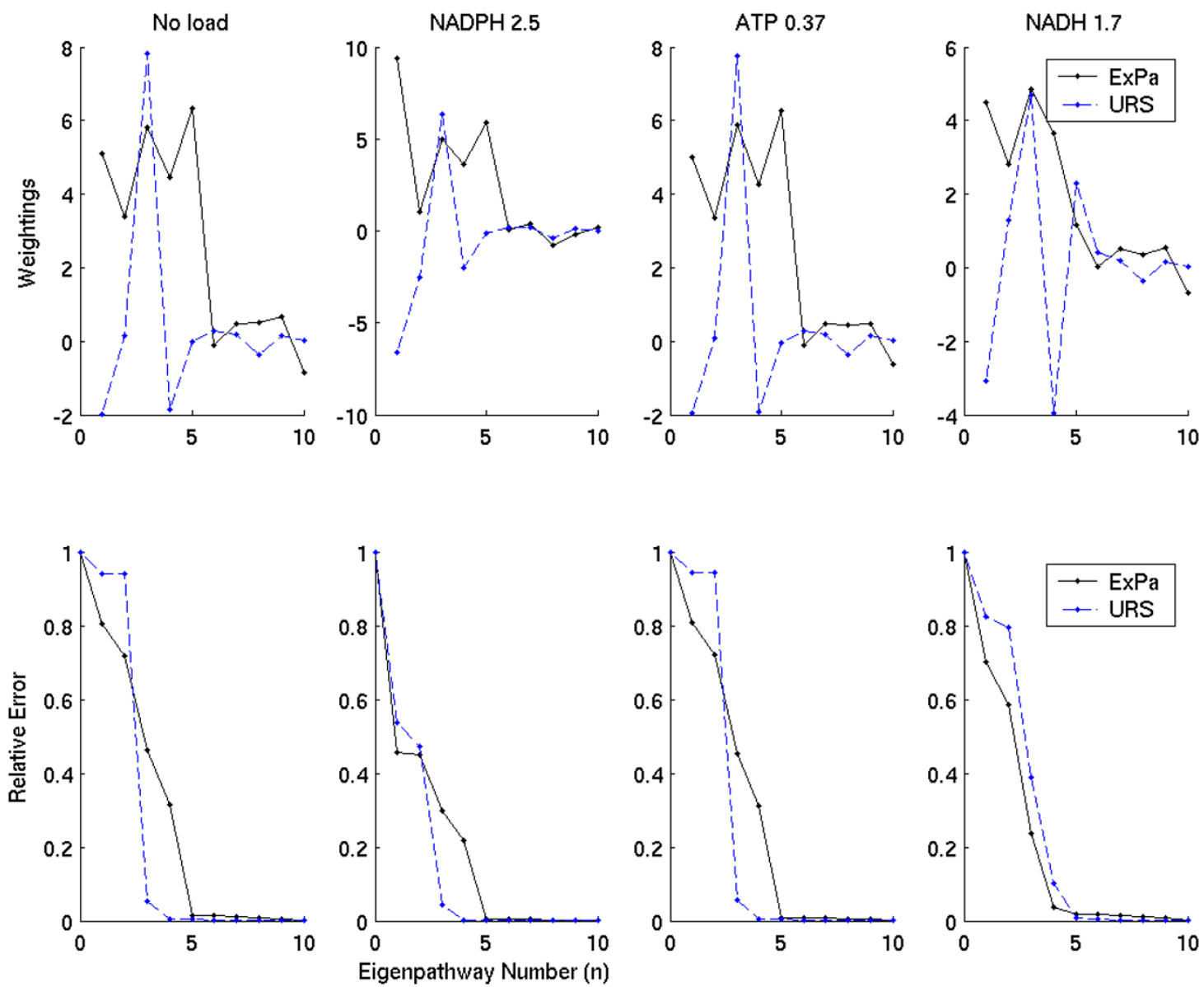

Figure 7

A comparison of how the eignpathways and PRMs reconstruct four different physiological flux distributions in the hRBC. The upper plots show the mode weightings for reconstructing the flux distributions, and the lower plots show the relative error between the reconstructed and physiological flux distributions for increasing numbers of modes. 


\section{Genome-scale sampling}

While the sampling approach detailed herein used the hRBC for demonstration purposes, the intended application for the method is genome-scale metabolic networks. A requirement for application to such networks is the demonstration of sufficient sampling of their much higher-dimensional steady-state solution spaces. In this section we demonstrate that sufficient sampling is feasible on the genome scale, in the same manner as was done for the hRBC in the Results section above. We use the bacterium Helicobacter pylori metabolic reconstruction [28], which is composed of 341 metabolic genes catalyzing 554 reactions amongst 485 metabolites. As can be seen in Figure 8 , satisfactory convergence is obtained for the first five modes after 150,000 samples.

\section{Discussion}

In previous work [24] it was demonstrated that SVD of the extreme pathway matrix for the hRBC metabolic network revealed the essential dimensionality of the regulatory problem. Furthermore, it was shown that the dominant features of the eigenpathways corresponding to the five largest singular values corresponded to control points in the hRBC metabolic network. These results are significant because they demonstrate a potentially powerful method for developing network-based understanding of regulation in genome-scale metabolic networks. Unfortunately, the method relies on the computation of the extreme pathways for these networks - a computational exercise that is currently infeasible. In an effort to circumvent this obstacle, we investigated the utility of randomly sampling
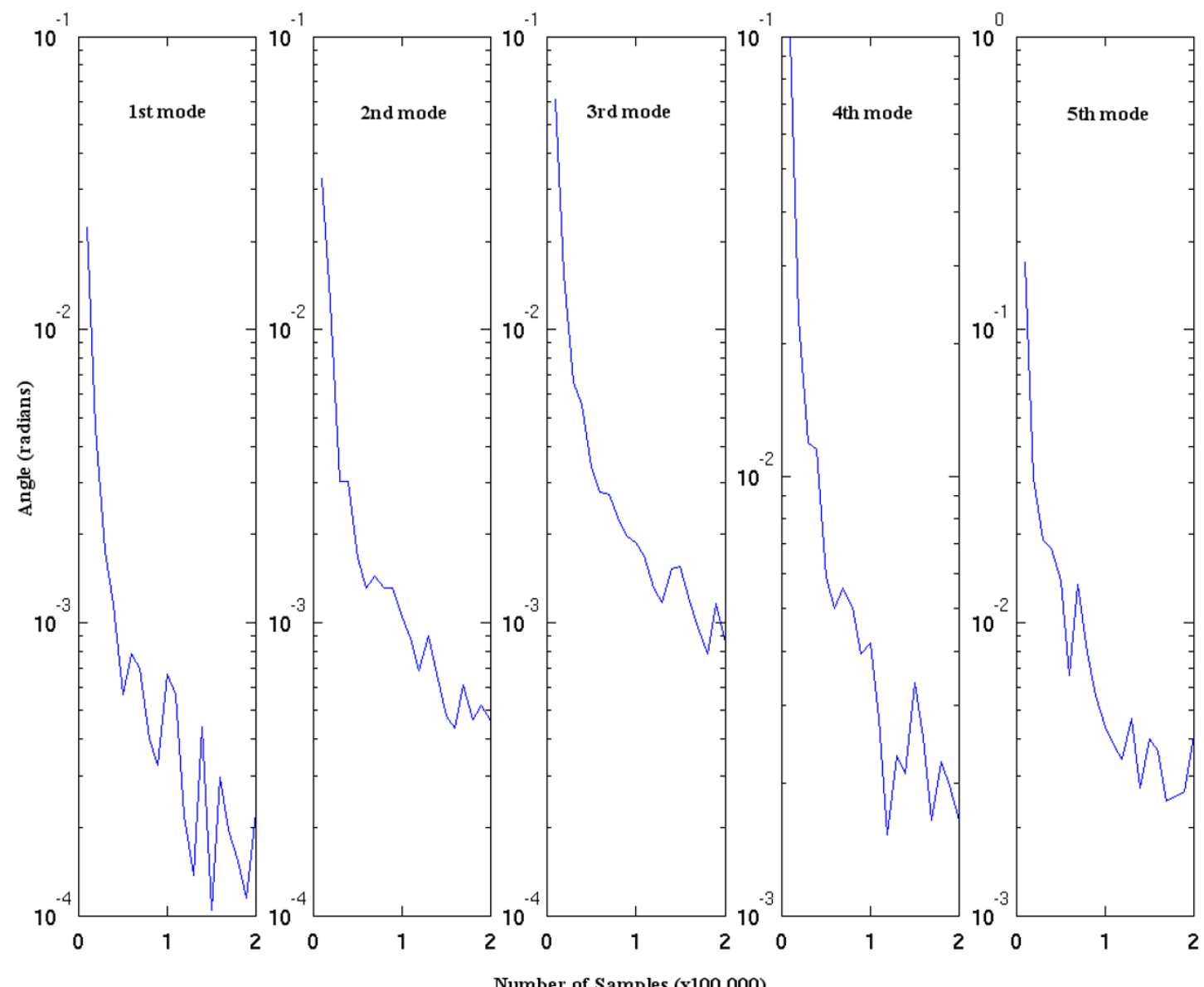

Number of Samples $(x 100,000)$

\section{Figure 8}

The SVD procedure was applied for increasingly larger sample sizes (up to 200,000 in increments of 10,000) for the metabolic network of the bacterium Helicobacter pylori and the first five modes at each sample size were recorded. These plots show the convergence of the angle between the modes for subsequent sample sizes. 
Table I: Metabolite abbreviations used in Figures 2-6.

\begin{tabular}{llll}
\hline Abbr. & Metabolite & Abbr. & Metabolite \\
\hline I3DPG & I,3-diphosphoglycerate & GLU & glucose \\
23DPG & 2,3-diphosphoglycerate & HX & hypoxanthine \\
2PG & 2-phosphoglycerate & IMP & inosine monophosphate \\
3PG & 3-phosphoglycerate & INO & inosine \\
6PGC & phosphogluconate & LAC & lactate \\
6PGL & phosphogluco-lactone & PEP & phosphenolpyruvate \\
ADE & Adenine & Pi & inorganic phosphate \\
ADO & Adenosine & PRPP & 5-phosphoribosyl-I-pyrophosphate \\
DHAP & dihydroxyacetone phosphate & PYR & Pyruvate \\
E4P & erythrose-4-phosphate & RIP & ribose-I-phosphate \\
F6P & fructose-6-phosphate & R5P & ribose-5-phosphate \\
FDP & fructose-I,6-bisphosphate & RL5P & ribulose-5-phosphate \\
G6P & glucose-6-phosphate & S7P & sedoheptulose-7-phosphate \\
GA3P & glyceraldehyde-3-phosphate & X5P & xylulose-5-phosphate \\
\hline
\end{tabular}

the steady-state solution space of the hRBC metabolic network, and through SVD of the matrix of sampled solution points arrived at comparable results to those obtained via SVD of $\mathbf{P}$. We present three main results. First, we found that the sampling procedure did lead to the same estimates of the dimensionality of the regulatory problem for the metabolic network studied. Secondly, we demonstrated that the resulting PRMs reconstruct physiological flux distributions as efficiently as did the eigenpathways. Thirdly, we showed that the flux adjustments represented by the PRMs were similar, and similarly interpretable, as those represented by the eigenpathways. In particular, we found that the ranking of reactions by their regulatory "importance" in the two approaches was highly correlated with high statistical significance.

It is important to note that there is no a priori reason for believing that SVD of $\mathbf{P}$ would be superior to SVD of $\mathbf{W}$. Thus, while the focus of this work was to explain the results of SVD of $\mathrm{W}$ in the context of what had already been shown for SVD of $\mathbf{P}$, it is not necessary that an effort be made to force SVD of $\mathbf{W}$ to reproduce SVD of $\mathbf{P}$. Instead, what was shown herein is that the approaches can be thought of as attacking the same problem and that SVD of $\mathrm{W}$ provides a way forward into genome-scale matrices to the line of inquiry that was begun with SVD of $P$, since sampling of genome-scale metabolic states has been accomplished at the genome-scale for E. coli and H. pylori $[29,30]$ - whereas the computation of $\mathbf{P}$ for these networks has proven infeasible. The results of the two methods will not generally be identical. In the case of SVD of $\mathbf{P}$ not only the shape of the space, but, more precisely, the distribution of the pathways along the edges of the space is what is being characterized. Because the samples are generated uniformly in the solution space, it is the shape of the solution space alone that is being characterized in this approach. Thus, the interpretation of SVD of $\mathrm{W}$ is more straightforward than is the interpretation of SVD of P.

We used physiological flux distributions computed from a kinetic model and compared how well PRMs and eigenpathways reconstructed them. As can be seen in Figure 7, the PRMs performed just as well, if not better, than the eigenpathways. Indeed, in three instances, the PRMs arrived at essentially complete reconstructions utilizing fewer PRMs. Based on these results, we estimate the regulatory problem for the hRBC to be roughly five-dimensional - in agreement with the estimate based on the eigenpathways.

We compared the PRM and eigenpathway flux distributions in two ways. Figures 2, 3, 4, 5, 6 utilize a flux map of the hRBC and are graphical in manner. Table 3 offers a more concise comparison in that it quantifies the projections of the PRMs and eigenpathways onto one another. In comparing the first five flux maps for the PRMs and eigenpathway fluxes directly we found the first two PRMs and eigenpathways have similar flux distributions (Figures 2 and 3 and Table 3). In the remaining three cases (Figures 4, 5, 6), though, the flux distributions diverge. It can be seen, especially in Table 3, that eigenpathway 3 is more similar to PRM 4 than PRM3, eigenpathway 4 is most similar to PRM 5, and eigenpathway 5 is almost exactly between PRMs 4 and 5. The reason for the divergence of modes is likely due to non-uniform densities of ExPas in the solution space, which since the SVD is a least squares method would have more variation in certain regions than presumably would a randomly sampled space.

The flux maps in Figures 2, 3, 4, 5, 6 demonstrate additional satisfying correspondence between PRMs and eigenpathways. First, the principal singular mode (first 
Table 2: Reaction abbreviations used in Figures 2-6. The corresponding reaction stoichiometries are also included.

\begin{tabular}{|c|c|c|}
\hline Abbr. & Enzyme Name & Metabolic Reaction \\
\hline ADA & Adenosine deaminase & $\mathrm{ADO}+\mathrm{H} 2 \mathrm{O} \rightarrow \mathrm{INO}+\mathrm{NH} 3$ \\
\hline AdPRT & Adenine phosphoribosyl transferase & $\mathrm{PRPP}+\mathrm{ADE}+\mathrm{H} 2 \mathrm{O} \rightarrow \mathrm{AMP}+2 \mathrm{Pi}$ \\
\hline$A K$ & Adenosine kinase & $\mathrm{ADO}+\mathrm{ATP} \rightarrow \mathrm{ADP}+\mathrm{AMP}$ \\
\hline ALD & Aldolase & $\mathrm{FDP} \leftrightarrow \mathrm{GA} 3 \mathrm{P}+\mathrm{DHAP}$ \\
\hline AMPase & Adenosine monophosphate phosphohydrolase & $\mathrm{AMP}+\mathrm{H} 2 \mathrm{O} \rightarrow \mathrm{ADO}+\mathrm{Pi}+\mathrm{H}$ \\
\hline AMPDA & Adenosine monophosphate deaminase & $\mathrm{AMP}+\mathrm{H} 2 \mathrm{O} \rightarrow \mathrm{IMP}+\mathrm{NH} 3$ \\
\hline ApK & Adenylate Kinase & $2 \mathrm{ADP} \leftrightarrow \mathrm{ATP}+\mathrm{AMP}$ \\
\hline DPGase & Diphosphoglycerate phosphatase & $23 \mathrm{DPG}+\mathrm{H} 2 \mathrm{O} \rightarrow 3 \mathrm{PG}+\mathrm{Pi}$ \\
\hline DPGM & Diphosphoglyceromutase & 13DPG $\rightarrow$ 23DPG + H \\
\hline EN & Enolase & $2 \mathrm{PG} \leftrightarrow \mathrm{PEP}+\mathrm{H} 2 \mathrm{O}$ \\
\hline G6PDH & Glucose-6-phosphate dehydrogenase & $\mathrm{G} 6 \mathrm{P}+\mathrm{NADP} \rightarrow 6 \mathrm{PGL}+\mathrm{NADPH}+\mathrm{H}$ \\
\hline GAPDH & Glyceraldehyde phosphate dehydrogenase & $\mathrm{GA} 3 \mathrm{P}+\mathrm{NAD}+\mathrm{Pi} \leftrightarrow$ I3DPG + NADH + H \\
\hline HGPRT & Hypoxanthine guanine phosphoryl transferase & $\mathrm{PRPP}+\mathrm{HX}+\mathrm{H} 2 \mathrm{O} \rightarrow \mathrm{IMP}+2 \mathrm{Pi}$ \\
\hline $\mathrm{HK}$ & Hexokinase & $\mathrm{GLU}+\mathrm{ATP} \rightarrow \mathrm{G} 6 \mathrm{P}+\mathrm{ADP}+\mathrm{H}$ \\
\hline IMPase & Inosine monophosphatase & $\mathrm{IMP}+\mathrm{H} 2 \mathrm{O} \rightarrow \mathrm{INO}+\mathrm{Pi}+\mathrm{H}$ \\
\hline LD & Lactate dehydrogenase & $\mathrm{PYR}+\mathrm{NADH}+\mathrm{H} \leftrightarrow \mathrm{LAC}+\mathrm{NAD}$ \\
\hline PDGH & 6-phosphoglycononate dehydrogenase & $6 \mathrm{PGC}+\mathrm{NADP} \rightarrow \mathrm{RL} 5 \mathrm{P}+\mathrm{NADPH}+\mathrm{CO} 2$ \\
\hline PFK & Phosphofructokinase & $\mathrm{F} 6 \mathrm{P}+\mathrm{ATP} \rightarrow \mathrm{FDP}+\mathrm{ADP}+\mathrm{H}$ \\
\hline PGI & Phosphoglucoisomerase & $\mathrm{G} 6 \mathrm{P} \leftrightarrow \mathrm{F} 6 \mathrm{P}$ \\
\hline PGK & Phosphoglycerate kinase & $13 \mathrm{DPG}+\mathrm{ADP} \leftrightarrow 3 \mathrm{PG}+\mathrm{ATP}$ \\
\hline PGL & 6-phosphoglyconolactonase & $6 \mathrm{PGL}+\mathrm{H} 2 \mathrm{O} \leftrightarrow 6 \mathrm{PGC}+\mathrm{H}$ \\
\hline PGM & Phosphoglyceromutase & $3 \mathrm{PG} \leftrightarrow 2 \mathrm{PG}$ \\
\hline PK & Pyruvate kinase & $\mathrm{PEP}+\mathrm{ADP}+\mathrm{H} \rightarrow \mathrm{PYR}+\mathrm{ATP}$ \\
\hline PNPase & Purine nucleoside phosphorylase & $\mathrm{INO}+\mathrm{Pi} \leftrightarrow \mathrm{HX}+\mathrm{RIP}$ \\
\hline PRM & Phosphoribomutase & $\mathrm{RIP} \leftrightarrow \mathrm{R} 5 \mathrm{P}$ \\
\hline PRPPsyn & Phosphoribosyl pyrophosphate synthetase & $\mathrm{R} 5 \mathrm{P}+\mathrm{ATP} \rightarrow \mathrm{PRPP}+\mathrm{AMP}$ \\
\hline RPI & Ribose-5-phosphate isomerase & $\mathrm{RL} 5 \mathrm{P} \leftrightarrow \mathrm{R} 5 \mathrm{P}$ \\
\hline TA & Transaldolase & $\mathrm{GA} 3 \mathrm{P}+\mathrm{S} 7 \mathrm{P} \leftrightarrow \mathrm{E} 4 \mathrm{P}+\mathrm{F} 6 \mathrm{P}$ \\
\hline TKI & Transketolase & $X 5 P+R 5 P \leftrightarrow S 7 P+E 4 P$ \\
\hline TK2 & Transketolase & $\mathrm{X} 5 \mathrm{P}+\mathrm{E} 4 \mathrm{P} \leftrightarrow \mathrm{F} 6 \mathrm{P}+\mathrm{GA} 3 \mathrm{P}$ \\
\hline TPI & Triose phosphate isomerase & $\mathrm{DHAP} \leftrightarrow \mathrm{GA} 3 \mathrm{P}$ \\
\hline XPI & Xylulose-5-phosphate epimerase & $\mathrm{RL} 5 \mathrm{P} \leftrightarrow \mathrm{X} 5 \mathrm{P}$ \\
\hline
\end{tabular}

PRM) is a valid flux distribution, adhering to mass balance and thermodynamic constraints just as is the case with the principal eigenpathway. Second, the regulatory correspondence that was achieved with the eigenpathways is also possible with the PRMs. For example, the dominant feature of the second PRM is also the flux split between glycolysis and the pentose phosphate pathway. The third singular mode describes, similarly to the third eigenpathway, the glycolytic pathway with the production of ATP, but instead of proceeding through to pyruvate with the production of NADH, it proceeds through to lactate with the utilization of NADH to produce NAD. The fourth PRM, as can be seen in Figure 5, is closer to eigenpathway 5, and this is due to the dominant feature of pyruvate uptake and subsequent conversion to lactate. Finally, the fifth PRM shares the same dominant characterization as eigenpathway 4, this being the flux split between lower glycolysis and the Rapoport-Luebering shunt.
The PRMs of $\mathrm{W}$ have a slightly different interpretation than the eigenpathways derived from P. Mathematically, the modes of $\mathbf{W}$ are the orthogonal directions of maximal variance in the steady-state solution space of the stoichiometric matrix. Biochemically, they correspond to the directions of greatest flexibility in achieving a networkwide flux distribution. The unequal variances in these orthogonal directions imply a hierarchy, wherein control of the more dominant modes gives the cell more control of achieving a desired flux distribution.

\section{Conclusion}

From these results we conclude that SVD of a sample of uniformly obtained steady state flux distributions for a metabolic network leads to a similar means of characterizing the regulatory problem as was obtained previously with ExPas. This was a necessary demonstration on a small system, before applying the technique to genomescale networks. With a number of genome-scale metabolic 
Table 3: The fractional projection of eigenpathways and PRMs onto one another.

\begin{tabular}{|c|c|c|c|c|c|c|c|c|c|c|}
\hline fromlonto & eigpath I & eigpath2 & eigpath3 & eigpath4 & eigpath5 & PRMI & PRM2 & PRM3 & PRM4 & PRM5 \\
\hline eigpath I & 0 & 0.021 & 0.021 & 0.126 & 0.384 & 0.950 & 0.107 & 0.077 & 0.247 & 0.054 \\
\hline eigpath2 & 0.022 & 0 & 0.039 & 0.187 & 0.300 & 0.028 & 0.983 & 0.016 & 0.195 & 0.168 \\
\hline eigpath3 & 0.025 & 0.045 & 0 & 0.011 & 0.252 & 0.212 & 0.189 & 0.671 & 0.899 & 0.231 \\
\hline eigpath4 & 0.114 & 0.165 & 0.009 & 0 & 0.108 & 0.049 & 0.029 & 0.280 & 0.378 & 0.763 \\
\hline eigpath5 & 0.387 & 0.295 & 0.214 & 0.120 & 0 & 0.282 & 0.284 & 0.430 & 0.584 & 0.560 \\
\hline PRMI & 0.957 & 0.027 & 0.180 & 0.055 & 0.282 & 0 & 0 & 0 & 0 & 0 \\
\hline PRM2 & 0.108 & 0.966 & 0.160 & 0.033 & 0.284 & 0 & 0 & 0 & 0 & 0 \\
\hline PRM3 & 0.078 & 0.016 & 0.569 & 0.311 & 0.430 & 0 & 0 & 0 & 0 & 0 \\
\hline PRM4 & 0.248 & 0.192 & 0.761 & 0.421 & 0.584 & 0 & 0 & 0 & 0 & 0 \\
\hline PRM5 & 0.055 & 0.165 & 0.195 & 0.848 & 0.560 & 0 & 0 & 0 & 0 & 0 \\
\hline
\end{tabular}

networks now significantly reconstructed for a number of organisms, the results presented in this work indicate that it should now be possible - given that feasible genomescale sampling has been demonstrated $[29,30]$ - to realize the dimensionality and mechanisms of metabolic regulation implicit in the topology of these networks.

\section{Methods}

\section{Human red blood cell model}

For this work we utilized a previously-constructed kinetic model [31] and a stoichiometric model $[22,24]$ of the human red blood cell. The stoichiometric model accounts for 39 metabolites and 32 internal metabolic reactions, and also contains 12 primary exchange fluxes and 7 currency exchange fluxes.

\section{Physiologic steady-state flux distributions}

Four steady-state flux distributions were calculated using an in silico kinetic model [31], as detailed in earlier work [24], and used to represent physiological states of interest in the solution space. The first distribution was for an unstressed state or normal physiological state, corresponding to no metabolic load. The other three corresponded to the primary metabolic demands placed upon the hRBC based on its physiologic role in the human body. These demands were for ATP (energy load), NADPH (oxidative load), and NADH (met-hemoglobin load). In each case, the load was maximized in the kinetic model to the highest value possible where the model could still be solved. Thus, they represented as high a load as the network could handle. All these states were previously computed and used in a previous study [24]. These maximum values were $0.37 \mathrm{mM} / \mathrm{h}$ for ATP load (above a mandatory $\sim 1 \mathrm{mM} / \mathrm{hr}$ running of the $\mathrm{Na}^{+} / \mathrm{K}^{+}$pump), 2.5 $\mathrm{mM} / \mathrm{h}$ for the NADPH load, and $1.7 \mathrm{mM} / \mathrm{h}$ for the NADH load.

\section{Physiologic flux constraints}

The null space of the stoichiometric matrix of the hRBC metabolic network is a (mathematically) unbounded space. To transform this space into one that is physiologi- cally relevant, we applied, as before, constraints by bounding the minimum and maximum fluxes for each reaction. All irreversible reactions were constrained with a minimum flux of $0 \mathrm{mM} / \mathrm{h}$ and a maximum flux of 2000 $\mathrm{mM} / \mathrm{h}$ unless experimentally-determined maximum flux values were available. Such maximum reaction rates were available for 22 reactions, and have been published previously $[22,32]$. Additionally, certain fluxes must have a minimum, non-zero value in order to sustain the hRBC. These values were obtained from a previous study on the hRBC $[32,33]$, and since they are few in number are given here: DPGase, $0.3 \mathrm{mM} / \mathrm{h}$; ATP production, $1 \mathrm{mM} / \mathrm{h}$; NADH production, $0.4 \mathrm{mM} / \mathrm{h}$; and NADPH production, $0.05 \mathrm{mM} / \mathrm{h}$.

\section{Hit-and-Run sampling of the solution space}

We use a variant of an artificial centering hit-and-run algorithm [34] to sample random flux distributions in the steady-state solution space of the hRBC. The algorithm is a random walk algorithm entailing three steps. The first step is to generate a set of "warmup" points in the solution space. This generation of warmup points was performed by slightly reducing the maximum flux and increasing the minimum flux for all reactions and then calculating a candidate flux distribution along a reaction coordinate using linear programming. Ten thousand such warmup points were created. The second step of the algorithm is to select an initial point from one of the "warm up" points generated. The warm up points were stored in a matrix $\mathbf{W}$ and were used to calculate an approximate center $s$ of the solution space. The third step calculates the sample points. Beginning with an initial, randomly selected point $x_{m}$ from $\mathrm{W}$, a direction vector is constructed by choosing a randomly selected point $y$ from $\mathrm{W}$ and moving along the direction vector $\mathbf{d}=\mathbf{y}$-s from $x_{m}$ resulting in a new point $x_{m+1}$. After each such move $x_{m+1}$ was randomly substituted into $\mathrm{W}$ and the approximate center recalculated. For the results presented herein, we saved a sample point every 1000 moves in the space. It should be noted that the red blood cell metabolic network does not have any non-trivial biochemical loops (type III ExPas) [35], and thus the 
samples generated do not violate "loop law" thermodynamic constraints [36,37] that would otherwise need to be explicitly addressed in sampling [30]. Note that in previous work [22], it was stated that the hRBC contains 16 Type III ExPas. The computation of ExPas in that work entailed the decoupling of reversible reactions into forward and backward reactions, and these resulting trivial loops were the 16 Type III pathways. In this work, reversible reactions were not decoupled, and so trivial Type III pathways were not an issue. One issue not encountered in this work which will is important for sampling genomescale networks is the issue of non-trivial Type III ExPas (more than 2 reactions). Since Type III ExPas correspond to thermodynamically infeasible flux distributions, the generation of samples from genome-scale systems will need to be cognizant of this issue. This issue has been addressed elsewhere and will not be an impediment to sampling genome-scale metabolic networks [30].

\section{Extreme pathways}

The extreme pathways were calculated from the stoichiometric matrix of the hRBC using a previously published algorithm [2]. $\mathrm{V}_{\max }$ values for the reactions in the hRBC have been previously published [22,32]. Each extreme pathway was scaled by the most limiting $\mathrm{V}_{\max }$ amongst all reactions that it contained. All of the extreme pathways had at least one reaction with an experimentally defined $\mathrm{V}_{\max }$.

\section{Angle between vectors}

Angles between modes were computed using standard linear algebra. For two vectors $\mathbf{u}$ and $\mathbf{v}$, the angle was computed as $\Theta=\cos ^{-1}(\mathbf{u} \cdot \mathbf{v} /\|\mathbf{u}\|\|\mathbf{v}\|)$.

\section{Regulatory importance of reactions}

The result of applying the SVD to either the matrix of ExPas or the matrix of uniformly sampled flux distributions is a set of eigenvectors. Each reaction has a corresponding loading vector, which is the vector of the reaction's contribution to each of the eigenvectors. Specifically, if $v_{i j}$ is the contribution of reaction $j$ to eigenvector $i$, and there are $m$ eigenvectors, then the loading vector for reaction $j$ is $\mathbf{l}_{j}=\left(\mathrm{v}_{1 j}, \mathrm{v}_{2 j}, \ldots, \mathrm{v}_{m j}\right)$. The score for reaction $j$ is then given by the $l^{2}$-norm of the loading vector: $\left|\mathbf{l}_{j}\right|=\left(\mathrm{v}_{1 j}{ }^{2}\right.$ $\left.+\mathrm{v}_{2 j}{ }^{2}+\ldots+\mathrm{v}_{m j}{ }^{2}\right)^{1 / 2}$.

\section{Abbreviations}

SVD, singular value decomposition; ExPa, extreme pathway; PRM, principal regulatory mode

\section{Authors' contributions}

CB and NP performed the sampling and interpretation of the results. CB drafted the manuscript and NP and BP provided critical edits.

\section{Acknowledgements}

This work was funded in part by NIHGMS grant number GM068837. BOP serves on the Scientific Advisory Board of Genomatica Inc.

\section{References}

I. Reed JL, Famili I, Thiele I, Palsson BO: Towards multidimensional genome annotation. Nat Rev Genet 2006, 7:|30- I4I.

2. Schilling $\mathrm{CH}$, Letscher D, Palsson BO: Theory for the systemic definition of metabolic pathways and their use in interpreting metabolic function from a pathway-oriented perspective. J Theor Biol 2000, 203:229-248.

3. Schilling $\mathrm{CH}$, Palsson $\mathrm{BO}$ : The underlying pathway structure of biochemical reaction networks. Proc Natl Acad Sci U S A 1998, 95:4193-4198.

4. Price ND, Reed JL, Papin JA, Famili I, Palsson BO: Analysis of metabolic capabilities using singular value decomposition of extreme pathway matrices. Biophys J 2003, 84:794-804.

5. Schuster S, Fell DA, Dandekar T: A general definition of metabolic pathways useful for systematic organization and analysis of complex metabolic networks. Nat Biotechnol 2000, I8:326-332.

6. Schuster S, Dandekar T, Fell DA: Detection of elementary flux modes in biochemical networks: a promising tool for pathway analysis and metabolic engineering. Trends Biotechnol 1999, I7:53-60.

7. Schuster S, Hilgetag C: On elementary flux modes in biochemical reaction systems at steady state. J Biol Syst 1994, 2: 165-182.

8. Papin JA, Reed JL, Palsson BO: Hierarchical thinking in network biology: the unbiased modularization of biochemical networks. Trends Biochem Sci 2004, 29:64I-647.

9. Schilling $\mathrm{CH}$, Schuster S, Palsson BO, Heinrich R: Metabolic pathway analysis: basic concepts and scientific applications in the post-genomic era. Biotechnol Prog 1999, I5:296-303.

10. Papin JA, Price ND, Wiback SJ, Fell DA, Palsson BO: Metabolic pathways in the post-genome era. Trends Biochem Sci 2003, 28:250-258.

II. Stelling J, Klamt S, Bettenbrock K, Schuster S, Gilles ED: Metabolic network structure determines key aspects of functionality and regulation. Nature 2002, 420:190-193.

12. Price ND, Papin JA, Palsson BO: Determination of Redundancy and Systems Properties of Helicobacter pylori's Metabolic Network Using Genome-Scale Extreme Pathway Analysis. Genome Res 2002, I 2:760-769.

13. Liao JC, Hou SY, Chao YP: Pathway analysis, engineering and physiological considerations for redirecting central metabolism. Biotechnol Bioeng 1996, 52:129-140.

14. Carlson R, Fell D, Srienc F: Metabolic pathway analysis of a recombinant yeast for rational strain development. Biotechnol Bioeng 2002, 79:121-134.

15. Pfeiffer T, I SV, Nuno JC, Montero F, Schuster S: METATOOL: for studying metabolic networks. Bioinformatics 1999, 15:25I-257.

16. Schuster S, Fell DA, Pfeiffer T, Dandekar T, Bork P: Elementary Mode Analysis Illustrated with Human Red Cell Metabolism. In BioThermoKinetics in the Post Genomic Era Edited by: Larsson D, Pahlman IL and Gustaffson L. Goeteborg, Chalmers; 1998:332-339.

17. Reed JL, Palsson BO: Genome-Scale In Silico Models of E. coli Have Multiple Equivalent Phenotypic States: Assessment of Correlated Reaction Subsets That Comprise Network States. Genome Res 2004, 14:1797-1805.

18. Papin JA, Price ND, Palsson BO: Extreme pathway lengths and reaction participation in genome-scale metabolic networks. Genome Res 2002, I 2:1889-1900.

19. Schilling $\mathrm{CH}$, Edwards JS, Letscher D, Palsson BO: Combining pathway analysis with flux balance analysis for the comprehensive study of metabolic systems. Biotechnol Bioeng 2000, 7I:286-306.

20. Famili I, Palsson BO: Systemic metabolic reactions are obtained by singular value decomposition of genome-scale stoichiometric matrices. Journal of Theoretical Biology 2003, 224:87-96.

21. Schilling CH, Palsson BO: Assessment of the Metabolic Capabilities of Haemophilus influenzae Rd through a Genome-scale Pathway Analysis. J Theor Biol 2000, 203:249-283.

22. Wiback SJ, Palsson BO: Extreme pathway analysis of human red blood cell metabolism. Biophys J 2002, 83:808-818. 
23. Schuster S, Pfeiffer T, Moldenhauer F, Koch I, Dandekar T: Exploring the pathway structure of metabolism: decomposition into subnetworks and application to Mycoplasma pneumoniae. Bioinformatics 2002, | 8:35I-36|.

24. Price ND, Reed JL, Papin JA, Wiback SJ, Palsson BO: Networkbased Analysis of Metabolic Regulation in the Human Red Blood Cell. Journal of Theoretical Biology 2003, 225: I85-194.

25. Golub GH, Kahan W: Calculating the Singular Values and Pseudoinverse of a Matrix. J of the Society for Industrial and Mathematics: Series B, Numerical Analysis 1965, 2:205-224.

26. Yoon H, Yang K, C. S: Feature Subset Selection and Feature Ranking for Multivariate Time Series. IEEE Transactions on Knowledge and Data Engineering 2005, 17:

27. Kendall M, Gibbons JD: Rank Correlation Methods. 5th 1990.

28. Thiele I, Vo TD, Price ND, Palsson B: An Expanded Metabolic Reconstruction of Helicobacter pylori (ilT34I GSM/GPR): An in silico genome-scale characterization of single and double deletion mutants. J Bacteriol 2005, 187:58I8-5830.

29. Almaas E, Kovacs B, Vicsek T, Oltvai ZN, Barabasi AL: Global organization of metabolic fluxes in the bacterium Escherichia coli. Nature 2004, 427:839-843.

30. Price ND, Thiele I, B.O. P: Candidate states of Helicobacter pylori's genome-scale metabolic network upon application of loop law thermodynamic constraints. Biophsyical Journal 2006, accepted:.

31. Jamshidi N, Edwards JS, Fahland T, Church GM, Palsson BO: Dynamic simulation of the human red blood cell metabolic network. Bioinformatics 200I, 17:286-287.

32. Joshi A, Palsson BO: Metabolic dynamics in the human red cell. Part III--Metabolic reaction rates. J theor biol 1990, 142:41-68.

33. Price ND, Schellenberger J, Palsson BO: Uniform Sampling of Steady State Flux Spaces: Means to Design Experiments and to Interpret Enzymopathies. Biophysical Journal 2004, 87:2172-2i 86

34. Kaufman DE, Smith RL: Direction Choice for Accelerated Convergence in Hit-and-Run Sampling. Operations Research 1998 , Jan-Feb:.

35. Price ND, Famili I, Beard DA, Palsson BO: Extreme pathways and Kirchhoff's second law. Biophys J 2002, 83:2879-2882.

36. Beard DA, Liang SD, Qian $\mathrm{H}$ : Energy balance for analysis of complex metabolic networks. Biophys J 2002, 83:79-86.

37. Qian H, Beard DA: Thermodynamics of stoichiometric biochemical networks in living systems far from equilibrium. Biophys Chem 2005, I 1 4:213-220.

Publish with Biomed Central and every scientist can read your work free of charge

"BioMed Central will be the most significant development for disseminating the results of biomedical research in our lifetime. "

Sir Paul Nurse, Cancer Research UK

Your research papers will be:

- available free of charge to the entire biomedical community

- peer reviewed and published immediately upon acceptance

- cited in PubMed and archived on PubMed Central

- yours - you keep the copyright
BioMedcentral 